\title{
Current trends and controversies in the management of patients with splenic flexure tumours
}

\author{
Chan $\mathrm{DS}^{1, *}$, Shah $\mathrm{PR}^{2}$, Soanes $\mathrm{M}^{3}$ and Saklani $\mathrm{A}^{4}$ \\ ${ }^{1}$ Chan DS, Wrexham Maelor Hospital, Wrexham, UK \\ ${ }^{2}$ Shah PR, University of Glamorgan, Pontypridd, UK \\ ${ }^{3}$ Soanes M, Cardiff University, Cardiff, UK \\ ${ }^{4}$ Princess of Wales Hospital, Bridgend, Wales, UK
}

\begin{abstract}
Aim: There exists a variation in practice in the management of tumours around the splenic flexure. We aim to determine the current opinion regarding the management of these tumours. Methods: An anonymised 10-part online questionnaire was sent to all members of the Association of Coloproctology of Great Britain and Ireland (ACPGBI). Results: The response rate was 24\% (111/464) with approximately half of respondents performing laparoscopic surgery. Electively, an extended right hemicolectomy is the preferred option by $63 \%$ of respondents followed by left hemicolectomy $(23 \%)$ and segmental resection (14\%). The upper sigmoid and rectosigmoid is the preferred site of anastomosis by $90 \%$ and $10 \%$ of respondents respectively. There were no significant differences in the type of operations performed by surgeons who practice laparoscopic or open surgery $(\mathrm{p}=0.10)$. A hand-sewn end-to-end anastomosis is most commonly performed (51\%) followed by a stapled side-to-side (36\%) and a stapled end-to-end (13\%) technique. Extended right hemicolectomy is also the preferred option in obstructing tumours. Of surgeons who perform segmental resections, $27 \%$ perform an on-table lavage and $9 \%$ perform a defunctioning stoma. Internal herniation following laparoscopic resection was only reported by a handful of surgeons. Conclusion: Opinion and practice in the management of patients with tumours around the splenic flexure are divided. Further trials are indicated to determine the best practice.
\end{abstract}

Keywords: left hemicolectomy; splenic flexure tumours; extended right hemicolectomy

\section{Introduction}

Colorectal cancer (CRC) is the third most common cancer in the United Kingdom after breastand lung [1,2]. Tumours around the splenic flexure account for between $2-8 \%$ of CRC and are associated with a higher risk of obstruction [3, 4] compared to tumours at other sites. However, reports of a poorer prognosis of patients with these tumours have been equivocal [4-6]. The surgical management of CRC has traditionally been excision in continuity with its vascular supply along with its lymphatic drainage to reduce the chance of local recurrence [7]. However, the blood supply to the splenic flexure has been shown to be somewhat variable. Therefore, various extent of resections has been advocated for the management of these tumours including an extended right hemicolectomy [8], segmental resection and left hemicolectomy $[9,10]$.

The aim of this study was to determine the current opinion regarding the management of these tumours. This will highlight the variation in practice and the need for well powered randomized trials to determine best practice.

\section{Materials and methods}

An online 10 part anonymised questionnaire (Appendix 1) was sent by email to all existing members of the Association of Coloproctology of Great Britain and Ireland (ACPGBI) who are consultant colorectal surgeons or general surgeons with special interest in colorectal surgery. The questionnaire was developed following discussion and a pilot study involving regional consultant colorectal surgeons. It included issues related to the type

* Corresponding author: Chan DS, Wrexham Maelor Hospital, Wrexham, UK, Tel.: 44292 07477477; Fax: 441978297558; E-mail: dcsy23@gmail.com

Received 12 November 2012 Revised 7 December 2012 Accepted 16 December 2012 Published 2 January 2013

Citation: Chan DS, Shah PR, Soanes M and Saklani A (2013) Current trends and controversies in the management of patients with splenic flexure tumours. J Cancer Res Ther. 1: 8-10. doi:10.14312/20524994.2013-2

Copyright: (C) 2013 Chan DS, et al. This is an open-access article distributed under the terms of the Creative Commons Attribution License, which permits unrestricted use, distribution and reproduction in any medium, provided the original author and source are credited. 
and details of the operation performed in the elective and emergency setting, their experience, significant complications and viewpoint of the colorectal surgeons regarding the operation. Data was tabulated in Microsoft Excel ${ }^{\circledR}$ and SPSS 18 was used for statistical analysis. Categorical data were compared with the chi square test.

\section{Results}

Questionnaires were sent to 464 members of the ACPGBI of which 111 replied (24\%). Approximately half of respondents perform laparoscopic surgery. Electively, an extended right hemicolectomy is the preferred option by 70 respondents $(63 \%)$ followed by left hemicolectomy $(26,23 \%)$ and segmental resection $(15,14 \%)$. Surgeons who perform an extended right hemicolectomy vary in their practice with regards to the pedicle ligated. Of the 70 respondents who prefer an extended right hemicolectomy in patients with these tumours, $15(22 \%)$ ligate the left branch of middle colic artery, 24 (34\%) ligate the left branch of middle colic and left colic artery and 19 (27\%) would include ligating the inferior mesenteric vein.

The upper sigmoid and rectosigmoid is the preferred site of anastomosis by $90 \%$ and $10 \%$ of respondents respectively. There were no significant differences in the type of operations performed by surgeons who practice laparoscopic or open surgery $(\mathrm{p}=0.10)$. A hand-sewn end-to-end anastomosis is most commonly performed (51\%) followed by a stapled side-to-side (36\%) and a stapled end-to-end (13\%) technique. Extended right hemicolectomy is also the preferred option in obstructing tumours. Of surgeons who perform segmental resections, $27 \%$ perform an on-table lavage and $9 \%$ perform a defunctioning stoma. Internal herniation following laparoscopic resection was only reported by a handful of surgeons.

Fifty surgeons (45\%) consider these tumours to carry a worse prognosis compared to tumours at other sites with the main reason cited as delayed presentation by 42 respondents (79\%). The remainder of respondents considered segmental resections and lower pedicle ligations to be associated with a worse prognosis.

\section{Discussion}

The management of splenic flexure tumours remains controversial and opinion divided. Despite within reach of a flexible sigmoidoscope which is used increasingly, these tumours usually present at a locally advanced stage infiltrating surrounding structures like the abdominal wall, diaphragm, pancreas and spleen [11]. Almost half of surgeons who responded thought that patients presenting with such tumours have a poor prognosis due to either late presentations or inadequate lymphatic clearance related to segmental resection or lower pedicle ligation. They are therefore more inclined to perform an extended right hemicolectomy. There seems to be inconsistency in the extent of resection without any evidence base.
Studies investigating the prognosis of splenic flexure tumours have been conflicting. This could be attributed to the relatively small number of patients studied [4, 11]. However, two of the largest studies have shown that splenic flexure tumours have a poor prognosis [12, 13]. A German multi-centered observational study of 29,658 consecutive patients showed that the cancers arising from the caecum and splenic flexure have the highest proportion of UICC stage III and IV tumours and lymphatic invasion [12]. Similar results were reported in the Large Bowel Cancer Project involving 4292 patients. This study showed that carcinoma at the splenic flexure was associated with the highest risk of obstruction (49\%); postoperative cardiopulmonary complications (36\%); inhospital mortality (18\%); and the lowest age-adjusted 5 -year survival (28\%), even after curative resection (38\%). This survival disadvantage was seen even in those without obstruction. Moreover, it was not accounted for by differences in age, sex, Dukes' stage or tumour differentiation between the various sites as stratification by these variables failed to alter significance [5].

The blood supply to the splenic flexure is variable. It has been demonstrated to be supplied by the inferior mesenteric artery (IMA) via the left colic in $89 \%$ of cases and by the superior mesenteric artery via the middle colic in $11 \%$ [14]. In $22 \%$ of cases, the middle colic artery was noted to be missing. A transverse colectomy for splenic flexure carcinoma is advocated by Goligher [15], ligating both middle colic at its origin and the ascending branch of the left colic to remove those nodes most likely to be involved. It seems that the best operation for lymphatic drainage would be ligating the middle colic and left colic with a transverse colectomy. However, it is technically easier to perform a right hemicolectomy, which would cover the small chance of metastasis to ileocolic lymph nodes. There have been no trials comparing extended right hemicolectomy to left hemicolectomy and segmental resection but there have been anecdotal reports of isolated ileocolic and superior mesenteric lymph node involvementin splenicflexure tumors $[8,13]$. Arandomised controlled trial comparing left hemicolectomy with high ligation of the IMA with a rectosigmoid anastomosis to segmental resection, showed a non-significant increase in anastomotic leaks after a left hemicolectomy but no difference in survival following either procedure [9]. Based on these results and the observation of other oncologic rules, left segmental colectomy rather than left hemicolectomy may theoretically be performed under laparoscopy without compromising the oncologic outcome. Functional outcomes following segmental colectomy have also been shown to be better in the first post-operative year compared with left hemicolectomy [9].

An open extended right hemicolectomy is a preferred option amongst the members when compared to open left hemicolectomy which can be technically challenging operation. Inspite of this, there is no significant difference 
in members performing these operations via laparoscopic approach. This makes one think that theleft hemicolectomy is therefore increasing in popularity as the uptake of laparoscopic procedures increase. In this survey, there exists a variation in practice with regards to ligation of the vascular pedicle root (IMV/IMA) and anastomosis at the recto-sigmoid junction. Although this may be appropriate for descending colon tumors, for splenic flexure tumors there might be problems with length and tension based on middle colic pedicle.

The management of splenic flexure tumours is not standardised. Options vary between extended right hemicolectomy, left hemicolectomy and segmental resections. The reason for this variation in practice may be related to technical (extended right hemicolectomy easier to perform, stapled anastomoses), and oncological (lymph node metastasis vary due variation in lymphatic drainage). The incidence of these tumours could increase if the flexible sigmoidoscopy screening programme is implemented [16]. A trial comparing extended right hemicolectomy and left hemicolectomy is therefore indicated to determine the best practice in themanagement of these tumours.

\section{Conclusion}

Opinion and practice in the management of patients with tumours around the splenic flexure are divided. Further research is indicated to determine best practice.

\section{Conflict of interest}

The authors wish to express that they have no conflict of interest.

\section{Acknowledgements}

We acknowledge all the members of the ACPGBI for participating in this survey.

\section{References}

[1] Office of National Statistics (2010) Cancer registrations in England 2008. Vol. 2011.

[2] White C (2011) Cancer in Wales 1995-2009: A comprehensive report (Wales cancer surveillance and intelligence Unit). Public Health Wales.

[3] Toms J (2004) Cancerstats Monograph 2004. Cancer Research UK, London.

[4] Levien DH, Gibbons S, Begos D, Byrne DW (1991) Survival after resection of carcinoma of the splenic flexure. Dis Colon Rectum 34: 401-403.

[5] Aldridge MC, Phillips RK, Hittinger R, Fry JS, Fielding LP (1986) Influence of tumour site on presentation, management and subsequent outcome in large bowel cancer. Br J Surg 73: 663-670.

[6] Steffen C, Bokey EL, Chapuis PH (1987) Carcinoma of the splenic flexure. Dis Colon Rectum 30: 872-874.

[7] Jamieson JK, Dobson JF (1909) VII. Lymphatics of the Colon: With Special Reference to the Operative Treatment of Cancer of the Colon. Ann Surg 50: 1077-1090.

[8] Sadler GP, Gupta R, Foster ME (1992) Carcinoma of the splenic flexure--a case for extended right hemicolectomy? Postgrad Med J 68: 487.
[9] Rouffet F, Hay JM, Vacher B, Fingerhut A, Elhadad A, et al. (1994) Curative resection for left colonic carcinoma: Hemicolectomy vs. Segmental colectomy: A prospective, controlled, multicenter trial. Dis Colon Rectum 37: 651-659.

[10] Nyam DC, Leong AF, Ho YH, Seow-Choen F (1996) Curative resection for left colonic carcinoma: Hemicolectomy vs. Segmental colectomy: A prospective, controlled, multicenter trial. Dis Colon Rectum 39: 1000-1003.

[11] Nakagoe T, Sawai T, Tsuji T, Jibiki M, Ohbatake M, et al. (2001) Surgical treatment and subsequent outcome of patients with carcinoma of the splenic flexure. Surg Today 31: 204-209.

[12] Benedix F, Schmidt U, Mroczkowski P, Gastinger I, Lippert H, et al. (2011) Colon carcinoma--classification into right and left sided cancer or according to colonic subsite?--Analysis of 29,568 patients. Eur J Surg Oncol 37: 134-139.

[13] Kim CW, Shin US, Yu CS, Kim JC (2010) Clinicopathologic characteristics, surgical treatment and outcomes for splenic flexure colon cancer. Cancer Res Treat 42: 69-76.

[14] Griffiths JD (1956) Surgical anatomy of the blood supply of the distal colon. Ann R Coll Surg Engl 19: 241-256.

[15] Goligher JC (1980) Surgery of the Anus, Rectum and Colon. Bailliere Tindall, London.

[16] Atkin WS, Edwards R, Kralj-Hans I, Wooldrage K, Hart AR, et al. (2010) Once-only flexible sigmoidoscopy screening in prevention of colorectal cancer: a multicentre randomised controlled trial. Lancet 375: 1624-1633.

\section{Appendix 1}

Survey on the management of patients with splenic flexure / upper descending colon tumours

1. Are you practicing laparoscopic colorectal procedures for these tumours?

2. What operation would you perform?

a. Left hemicolectomy

b. Extended right hemicolectomy

c. Segmental resection

3. For obstructing left sided colonic carcinomas, your preference would be:

a. Segmental left colectomy

b. Extended right colectomy

c. Resection with stoma

d. Other

4. If you were to perform a segmental resection of the colon, would you perform

a. On table lavage

b. Covering stoma

5. Which blood vessels would you ligate? (select any of the below) a. Left colic artery

b. High inferior mesenteric vein with left colic artery

c. Left branch of middle colic artery

6. Where would you perform the anastomosis?

a. Upper sigmoid

b. Rectosigmoid

7. What type of anastomosis do you perform?

a. Hand sewn 'end to end'

b. Stapled 'side to side'

c. Stapled 'EEA gun'

8. Have you had an internal herniation following laparoscopic left hemicolectomy?

9. Do you think these tumours carry a worse prognosis?

10. If yes, what do you think the reasons are? (select any of the options)

a. Due to segmental resections only

b. Lower pedicle ligations

c. Delayed presentation / advanced disease 